\title{
New and improved diagnostics for detection of drug-resistant pulmonary tuberculosis
}

\author{
Justin O'Grady ${ }^{\mathrm{a}, \mathrm{b}}$, Markus Maeurer ${ }^{\mathrm{c}}$, Peter Mwaba ${ }^{\mathrm{b}, \mathrm{d}}$, Nathan Kapata ${ }^{\mathrm{b}, \mathrm{d}}$, \\ Matthew Bates ${ }^{a, b}$, Michael Hoelscher ${ }^{\mathrm{e}}$ and Alimuddin Zumla ${ }^{\mathrm{a}, \mathrm{b}}$
}

\begin{abstract}
aDepartment of Infection, University College London Medical School, Windeyer Institute of Medical Sciences, London, UK, ' University of Zambia and University College London Medical School (UNZAUCLMS) Research and Training Programme, University Teaching Hospital, Lusaka, Zambia ${ }^{\mathrm{C}}$ Department of Microbiology, Tumour and Cell Biology, Karolinska Institute, Stockholm, Sweden, dMinistry of Health, Lusaka, Zambia and ${ }^{e}$ Department for Infectious Diseases and Tropical Medicine, Klinikum of the University of Munich, Munich, Germany

Correspondence to Dr Justin O'Grady, Department of Infection, University College London Medical School, Windeyer Institute, 46 Cleveland Street, London W1T 4JF, UK

Tel: +44 2076799486; e-mail: j.ogrady@ucl.ac.uk
\end{abstract}

Current Opinion in Pulmonary Medicine 2011, 17:134-141

\section{Purpose of review}

Tuberculosis (TB) remains a global emergency and continues to kill 1.7 million people globally each year. Drug-resistant TB is now well established throughout the world and most TB patients are not being screened for drug resistance due to lack of laboratory resources and rapid accurate point-of-care tests. Accurate and rapid diagnosis of TB and drug-resistant TB is of paramount importance in establishing appropriate clinical management and infection control measures. During the past decade, there have been significant advances in diagnostic technologies for TB and drug-resistant TB. The purpose of this article is to review the current data, recommendations and evidence base for these tests.

\section{Recent findings}

Second-line drug susceptibility testing (DST) is complex and expensive. Automated liquid culture systems and molecular line probe assays are recommended by the WHO as the current 'gold standard' for first-line DST. Liquid culture DST for aminoglycosides, polypeptides and fluoroquinolones has been shown to have relatively good reliability and reproducibility for diagnosis of extensively drug-resistant TB; however, DST for other second-line drugs (ethionamide, prothionamide, cycloserine, terizidone, paraaminosalicylic acid, clofazimine, amoxicillin-clavulanate, clarithromycin, linezolid) is not recommended. Automated liquid culture systems are currently recommended by the WHO as the 'gold standard' for second-line DST.

\section{Summary}

In this review, we describe the phenotypic and genotypic methods currently available for the diagnosis of TB and drug-resistant forms of Mycobacterium tuberculosis and discuss future prospects for TB diagnostics. Current technologies for the detection of drug resistant $M$. tuberculosis vary greatly in terms of turnaround time, cost and complexity. Ultimately, the 'holy grail' diagnostic for TB must fulfil all technical specifications for a good point-of-care test, screen for drug resistance concurrently and be adaptable to the various health system levels and to countries with diverse economic status and TB burden.

\section{Keywords}

diagnostics, drug-resistant tuberculosis, review, tuberculosis

Curr Opin Pulm Med 17:134-141

C 2011 Wolters Kluwer Health | Lippincott Williams \& Wilkins 1070-5287

\section{Introduction}

Tuberculosis (TB) remains a global emergency and continues to kill 1.7 million people globally, each year. During the past decade, there have been significant advances in diagnostic technologies for TB. Increase in both public and private investment and the joint efforts of the World Health Organisation (WHO) and the Foundation for Innovative New Diagnostics (FIND) have facilitated this process $\left[1^{\bullet \bullet}\right]$. FIND are co-developing new diagnostics technologies that are targeted for use at three health system levels: reference laboratory, microscopy centre/peripheral laboratories and primary healthcare level/health post. Such new and improved diagnostics technologies are urgently required for the global fight against the TB epidemic. Liquid media for culture and drug susceptibility testing (DST), molecular line probe assays for screening people at risk of multiple drugresistant TB (MDR-TB), LED microscopy and noncommercial culture and DST have all been endorsed by the WHO since 2007 (Fig. 1). Endorsement of technology by the WHO combined with FIND's negotiations on pricing 
with industry has made new and improved diagnostics more affordable and feasible, for the first time, for developing countries $\left[1^{\bullet \bullet}\right]$.

Widespread incorrect use of isoniazid and rifampicin over the past decades has resulted in emergence and spread of MDR-TB and extensively drug-resistant TB (XDR-TB) globally. These difficult to treat, drug-resistant forms of TB are increasingly seen in Asia, eastern Europe, South America and sub-Saharan Africa, disrupting TB and HIV control programmes $\left[3,4,5^{\bullet}, 6,7^{\bullet \bullet}, 8^{\bullet \bullet}\right]$ (Fig. 2). Each year, an estimated half a million MDR-TB cases develop, of which only around $7 \%$ are diagnosed. In the 27 highburden MDR-TB countries, only $1 \%$ of new TB patients had DST performed in 2008 because of a lack of laboratory capacity $\left[8^{\bullet \bullet}, 9\right]$. As a result, patients with drugresistant TB may be inappropriately treated, drug-resistant Mycobacterium tuberculosis strains may spread in the community and amplification of resistance may occur $\left[10^{\bullet}\right]$. Accurate and rapid diagnosis of drug-resistant $\mathrm{TB}$ is of paramount importance in establishing appropriate clinical management and infection control measures $\left[1^{\bullet \bullet}, 7^{\bullet \bullet}\right]$.

The 'gold standard' method for M. tuberculosis DST is the indirect $1 \%$ proportion method. This method was developed in the 1960s and is still used in many laboratories, especially in developing countries, because it is inexpensive and easily accessible [11]. Because of the long turnaround time (weeks to months) associated with such conventional DST methods, several new approaches have been developed for faster detection of drug-resist-

\section{Key points}

- New and improved methods for the detection of drug-resistant tuberculosis (TB) are described in this review.

- Phenotypic M. tuberculosis drug susceptibility testing (DST) methods are inexpensive and accurate but time consuming.

- Genotypic M. tuberculosis DST methods are rapid and accurate but expensive.

- Second-line DST is complex and expensive.

- The various M. tuberculosis DST methods described are all currently required to suit the diverse economic status and TB burden of countries worldwide and to suit the different health system levels.

ant TB. These methods can be divided into two categories: culture-based or phenotypic methods and nucleic acid-based or genotypic methods.

DST for first-line anti-TB drugs is most accurate for rifampicin (RIF) and isoniazid (INH) and less reliable and reproducible for streptomycin, ethambutol (EMB) and pyrazinamide (PZA). RIF resistance is a valid and reliable indicator of MDR-TB [12]. Automated liquid culture systems and molecular line probe assays are recommended by the WHO as the current 'gold standard' for first-line DST. Second-line DST is complex and expensive. Liquid culture DST for aminoglycosides, polypeptides and fluoroquinolones has been shown to have relatively good reliability and reproducibility for diagnosis of XDR-TB; however, DST for other second-line drugs

Figure 1 The Stop-TB Partnership's new diagnostics working group tuberculosis diagnostics pipeline

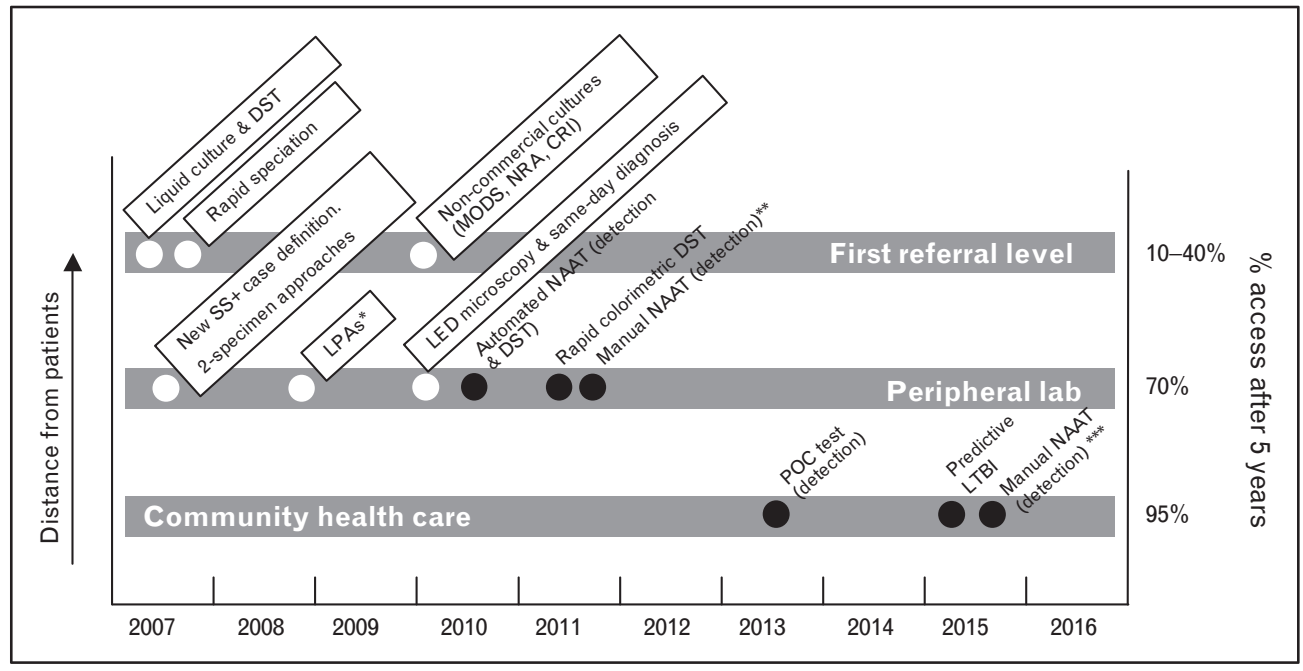

CRI, colorimetric redox indicator assay; DST, drug susceptibility test; LED, light-emitting diode; LPA, line probe assay; LTBI, latent tuberculosis infection; MODS, microscopic observation drug susceptibility; NAAT, nucleic acid amplification test; NRA, nitrate reductase assay; POC, point of care. ${ }^{*}$ Manual NAAT: technology for MTB drug susceptibility testing. ${ }^{* *}$ Manual NAAT: technology for MTB detection and peripheral laboratory. ${ }^{* * *}$ Manual NAAT: technology for MTB detection at the community healthcare level. Technologies in boxes: endorsed by WHO. Modified with permission from [2]. 


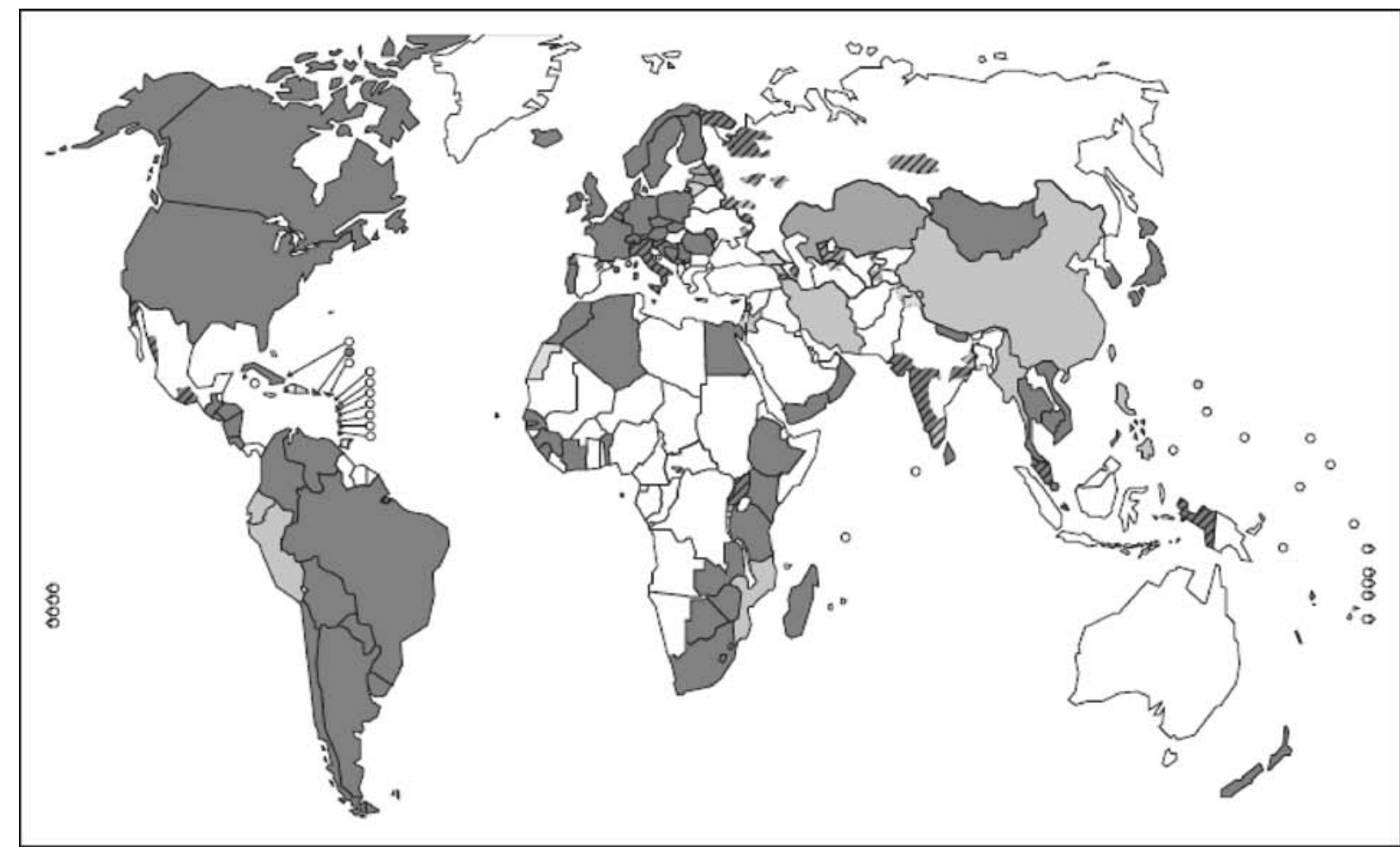

, 0 to $<3 ; \square, 3$ to $<6$; permission from [2].

(ethionamide, prothionamide, cycloserine, terizidone, para-aminosalicylic acid, clofazimine, amoxicillin-clavulanate, clarithromycin, linezolid) is not recommended [12]. Automated liquid culture systems are currently recommended by the WHO as the 'gold standard' for second-line DST $\left[12,13^{\bullet \bullet}, 14\right]$. In this review, we describe the phenotypic and genotypic methods currently available for the diagnosis of drug-resistant forms of $M$. tuberculosis and discuss future prospects for TB diagnostics.

\section{Definitions of drug-resistant tuberculosis}

MDR-TB is defined as resistance to the two key first-line anti-TB drugs, INH and RIF. XDR-TB is defined as TB caused by strains of $M$. tuberculosis resistant to at least INH and RIF (i.e. MDR-TB), plus any fluoroquinolone and at least one of three injectable drugs used in anti-TB treatment, capreomycin, kanamycin or amikacin $\left[7^{\bullet \bullet}, 8^{\bullet \bullet}\right]$.

\section{Phenotypic drug susceptibility testing}

Culture-based or phenotypic DST methods are accurate and inexpensive but are disadvantaged by relying on the growth on M. tuberculosis, rendering them time consuming [15]. Phenotypic DST methods are performed on solid or liquid media as direct or indirect tests. Direct methods are those used directly on patient samples where a set of drug-containing and drug-free media is inoculated directly with a patient specimen. Indirect DST involves inoculation of drug-containing media with a pure culture grown from the original patient specimen [12]. Commercial automated liquid culture DST methods have a relatively short turnaround time (because of sensitive automation and M. tuberculosis's relatively faster growth in liquid compared with solid media) and are highly accurate but are expensive and require specialist equipment $\left[16^{\circ}\right]$. The WHO considered evidence for the accuracy and role of a number of noncommercial culture-based methods that utilize widely available and inexpensive laboratory equipment and supplies and recommended selected methods as interim measures while capacity for automated culture DST and/or genotypic DST are being developed $\left[1^{\bullet \bullet}, 17^{\bullet \bullet}, 18\right]$. Microscopic observation drug susceptibility (MODS) $\left[17^{\bullet \bullet}, 19-21\right]$ and colorimetric redox indicator (CRI) $[22,23]$ methods and the nitrate reductase assay (NRA) $\left[15,24^{\bullet \bullet}, 25\right]$ received WHO approval $\left[17^{\bullet \bullet}\right]$. Such methods have similar accuracy to commercial liquid culture systems and could be implemented in high-burden, low-income settings with minimum cost; however, these tests require extensive operator training, standardization and quality assurance before implementation $\left[1^{\bullet \bullet}\right]$. 


\section{Commercial liquid culture drug susceptibility testing}

The most commonly used commercially available automated liquid culture DST system is the BACTEC MGIT 960 system with the BACTEC MGIT 960 SIRE kit (Becton Dickinson, Franklin Lakes, New Jersey, USA). This is an indirect qualitative method for the detection of first-line drug resistance to streptomycin, INH, RIF and EMB [16 $6^{\circ}$. The test, which is performed on $M$. tuberculosis complex (MTBC) positive culture, takes approximately $4-13$ days to obtain a result. The test was developed with critical concentrations for streptomycin $(1 \mu \mathrm{g} / \mathrm{ml})$, INH $(0.1 \mu \mathrm{g} / \mathrm{ml})$, RIF $(1 \mu \mathrm{g} /$ $\mathrm{ml})$ and $\operatorname{EMB}(5 \mu \mathrm{g} / \mathrm{ml})$ that are slightly lower than the critical concentrations used in the proportion method test, in order to avoid false susceptibility results. A PZA DST kit is also available for the MGIT system. The test is based on growth of the MTBC isolate in a drug-containing tube compared with a drug-free tube (growth control). Continuous analysis of fluorescence by the BACTEC MGIT 960 instrument in the drug-containing tube compared with the fluorescence of the growth control tube is used to determine susceptibility results. The instrument automatically interprets these results and reports a susceptible or resistant result $\left[16^{\bullet}, 26\right]$. This method has been demonstrated to be equivalent to the proportion method standard and has been endorsed by the WHO $\left[27,28,29^{\bullet}\right]$. Other nonradiometric automated liquid culture systems capable of $M$. tuberculosis DST include the BacT/ALERT MB (bioMerieux Inc., Durham, North Carolina, USA) system and the VersaTREK system (Trek Diagnostic Systems, West Lake, Ohio, USA) $[28,30]$.

\section{Microscopic observation drug susceptibility}

MODS is a liquid culture-based test that can detect members of the MTBC in sputum and can assess INH and RIF susceptibility either directly on sputum samples or indirectly on $M$. tuberculosis isolates. This method utilizes the markedly faster growth of $M$. tuberculosis in liquid media than on solid media and the characteristic microscopic cording appearance of $M$. tuberculosis in liquid media. Cultures containing supplemented Middlebrook 7H9 medium are microscopically examined for microcolonies which can be detected in a median of 7 days. INH and RIF can be incorporated in the testing process to enable MDR-TB detection [21]. Drug-free and drugcontaining media are inoculated with specimens from patients or M. tuberculosis isolates, and cultures are microscopically examined. Growth of $M$. tuberculosis in drugfree media indicates a positive culture, whereas growth of $M$. tuberculosis in both drug-free and drug-containing media indicates resistance $\left[17^{\bullet \bullet}, 19,21\right]$.
Meta-analysis of direct and combined (direct and indirect) testing of MODS performed by the WHO indicates that the method is $98 \%$ sensitive and $99 \%$ specific for the detection of RIF resistance and $91 \%$ sensitive for INH resistance [18]. High sensitivity and specificity are retained in direct MODS testing. The capability to perform MODS DST directly on sputum samples in a short timescale and the low cost of reagents are advantages of this technique [21], but the WHO only endorses its implementation at reference laboratory level [18].

\section{Colorimetric redox indicator methods}

The principal of CRI methods is the reduction of a coloured indicator which is added to the culture medium after cultured $M$. tuberculosis have been exposed to the test antibiotic [30]. Drug resistance is detected by a change in colour of the indicator, which is directly proportional to the number of viable mycobacteria remaining in the medium after exposure to the antibiotic. Different indicators have been evaluated giving comparable results in agreement with the proportion method reference standard. Among the different growth indicators used are the tetrazolium salts XTT and MTT and the redox indicators Alamar blue and resazurin [31].

Data analysis performed by the WHO showed that CRI methods are highly sensitive and specific for the detection of RIF and INH resistance (98 and $97 \%$ sensitive respectively; 99 and $98 \%$ specific, respectively) and these methods were endorsed by the WHO in 2010 [18]. CRI methods are indirect tests performed on $M$. tuberculosis isolates, therefore turnaround time to results is not faster than conventional phenotypic DST [18,31].

\section{Nitrate reductase assay}

The NRA, also known as the Griess method, is a simple technique based on the capacity of $M$. tuberculosis to reduce nitrate to nitrite. By incorporating $1 \mathrm{mg} / \mathrm{ml}$ potassium nitrate $\left(\mathrm{KNO}_{3}\right)$ in Lowenstein-Jensen medium, the reduction of nitrate can be detected using the Griess reagent, which produces a coloured reaction [11]. In the presence of antibiotic at the critical concentration, development of a red-pink colour in the medium represents resistance. Susceptible strains lose the capacity to reduce nitrate in the presence of the antibiotic, thus produce no colour. The use of nitrate reduction as an indicator of growth, before colonies can be seen macroscopically, reduces the turnaround time to results compared with conventional methods [11]. The WHO recommends [18] that the NRA be used as a direct test on smear-positive sputum specimens or as an indirect test on $M$. tuberculosis isolates grown from conventional solid culture. Data on combined (direct and indirect) use showed that the NRA is $97 \%$ sensitive and $100 \%$ specific 
for the detection of RIF resistance and $97 \%$ sensitive and 99\% specific for detection of INH resistance [18]. Diagnostic accuracy data for direct testing alone does not differ significantly. Reagents for NRA are nonproprietary and relatively inexpensive, but indirect testing using NRA is not faster than conventional phenotypic DST using solid media $[11,18]$.

Other new and existing phenotypic DST methods, which have not been endorsed by the WHO, are reviewed elsewhere [30] and include the slide-culture technique, mycobacteriophage-based methods including the Fas$t$ Plaque assay and luciferase reporter phages, the E-test, the thin-layer agar method and the TK medium.

\section{Genotypic drug susceptibility testing}

Several molecular diagnostics assays are commercially available for the detection of the MTBC, including the COBAS TaqMan MTB PCR test (Roche Diagnostics, Basel, Switzerland) [32*•], artus $M$. tuberculosis PCR kits (Qiagen, Hamburg, Germany) [33] and the Amplified Mycobacterium Tuberculosis Direct (AMTD) test (Gen-Probe Inc., San Diego, California, USA) [34 ${ }^{\circ}$. Recently, commercial and 'in-house' molecular diagnostics assays for the detection of the MTBC and the simultaneous detection of drug resistance have been described [35-40]. These methods are generally nucleic acid amplification tests (NAATs) and include hybridization assays (e.g. line probe assays), real-time PCR assays (often using molecular beacon probes) and sequencing assays. The most common mutations associated with drug resistance have been described and are publically available on the TB drug resistance mutation database [ $\left.41^{\bullet \bullet}\right]$. Genotypic DST methods target these well characterized resistance associated mutations to identify drug resistant $M$. tuberculosis. One of the most important drugs in the treatment of TB is RIF and RIF resistance is particularly suitable for genotypic DST because 95\% of RIF resistance associated mutations are present in an $81 \mathrm{bp}$ region of the $r p o B$ gene known as the rifampicin resistance determining region (RRDR) [36]. Molecular detection of resistance to other anti-TB drugs, such as INH and some second-line drugs, is more complex and requires detection of mutations in multiple genes for good correlation with phenotypic results. Accurate genotypic DST for first-line and second-line anti-TB drugs is, therefore, technically challenging. Improved multiplex-PCR technology and improved multianalyte detection technology will make genotypic DST a more powerful technique in future.

\section{Line probe assays}

Line probe assays were endorsed by the WHO in 2008 for molecular detection of drug resistance from smearpositive patients at risk of MDR-TB [42]. Two commer- cial LPAs are currently available: the INNO-LiPA Rif.TB test (Innogenetics NV, Gent, Belgium) and the GenoType MTBDRplus test (Hain Lifescience GmbH, Nehren, Germany) [29 $9^{\bullet}$. LPAs use a PCR/hybridization technique to identify members of the MTBC while simultaneously identifying drug-resistant strains by detecting the most common single nucleotide polymorphorisms (SNPs) associated with resistance. Metaanalyses have shown that LPAs are highly accurate for the detection of first-line drug resistance, especially in smear-positive sputum specimens $\left[29^{\bullet}, 39,43\right]$. WHO analysis of systematic reviews and meta-analyses showed that LPAs are highly sensitive $(\geq 97 \%)$ and specific $(\geq 99 \%)$ for the detection of RIF resistance, alone or in combination with INH (sensitivity $\geq 90 \%$; specificity $\geq 99 \%$ ), on isolates of $M$. tuberculosis and on smearpositive sputum specimens. Accuracy for detection of MDR-TB was 99\%, which remained unchanged when RIF resistance alone was used as a proxy marker for MDR-TB [42]. Hain Lifesciences released the GenoType MTBDRsl test in 2009, designed to test for resistance to second-line anti-TB drugs (fluoroquinolones, ethambutol, aminoglycosides and cyclic peptides), and which can be used in combination with the MTBDRplus test to identify XDR-TB [44-46]. The major advantage of LPAs is that they can be performed directly on smearpositive sputum samples, giving rapid (approximately $5 \mathrm{~h}$ ) drug susceptibility results without the need for culture. Many laboratories now use LPAs as the primary method for DST on cultured isolates of M. tuberculosis, replacing phenotypic DST [29 $9^{\circ}$. The disadvantages of LPAs are that they are labour intensive and require highly trained personnel and dedicated laboratory space and equipment [47].

\section{Xpert Mycobacterium tuberculosis/rifampicin assay}

One of the most promising new point-of-care (POC) diagnostics technologies to be developed in recent years is the GeneXpert (Cepheid, Sunnyvale, California, USA). The GeneXpert machine is a fully automated closed system that performs both sample preparation and realtime PCR, producing results in less than $2 \mathrm{~h}$ (Fig. 3). The Xpert MTB/RIF test, designed for the GeneXpert system, is capable of detecting the MTBC while simultaneously detecting RIF resistance (targeting the RRDR of the $r p o B$ gene). Analytical sensitivity and specificity is $100 \%$ according to a recent study using RIF resistant and sensitive $M$. tuberculosis isolates and nontuberculosis bacteria, fungi and viruses [36]. A clinical validation study of the method was performed using 107 clinical sputum samples from suspected TB cases in Vietnam. The Xpert MTB/RIF test detected 29/29 (100\% sensitivity) smear-positive culture-positive cases and 33/39 (84.6\% sensitivity) smear-negative culture-positive cases, 
Figure 3 Cepheid GeneXpert IV and Xpert MTB/RIF test cartridge

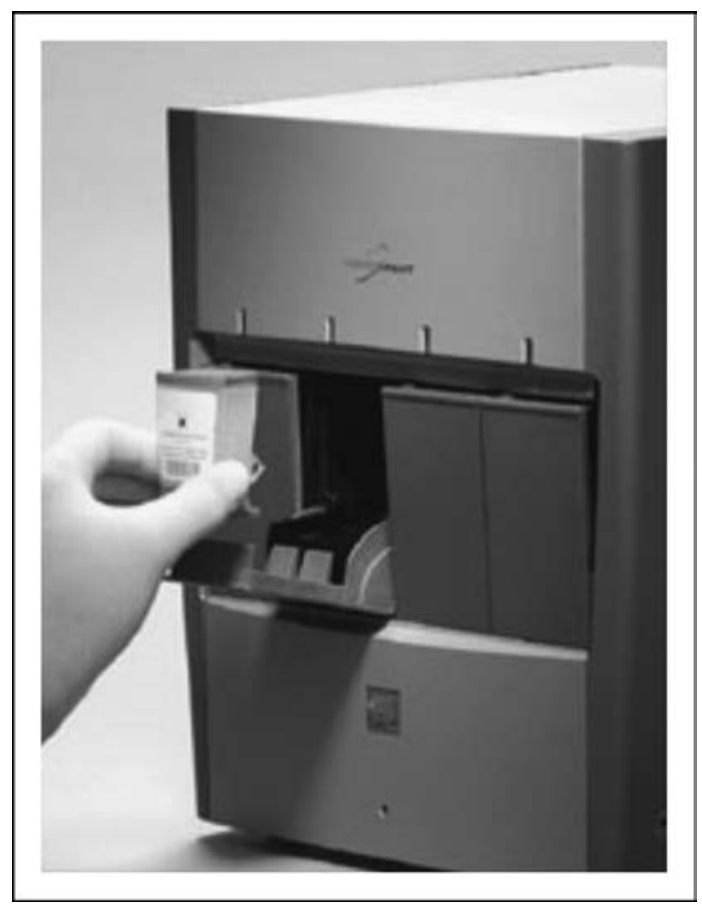

From Cepheid, reproduced with permission.

and M. tuberculosis was not detected in 25/25 $(100 \%$ specificity) of the culture-negative samples. In the same study, 64 smear-positive culture-positive sputa from patients previously treated for TB in Uganda were tested and the Xpert MTB/RIF test detected 63/64 (98.4\% sensitivity) culture-positive cases and nine of nine ( $100 \%$ sensitivity) cases of RIF resistance. RIF resistance was excluded in $55 / 55$ susceptible cases $-100 \%$ specificity $\left[48^{\bullet \bullet}\right.$ ]. In a recent multicentre (Peru, Azerbaijan, South Africa and India) evaluation study of 1730 patients with suspected drug-sensitive or multidrug-resistant pulmonary TB published in the New England Journal of Medicine, a single Xpert MTB/RIF test was 98.2 and $72.5 \%$ sensitive on smear-positive $(n=561)$ and smearnegative $(n=171)$ TB patients, respectively, and the test was $99.2 \%$ specific [ $49^{\circ}$ ]. Compared to phenotypic DST, the MTB/RIF test correctly identified 200 of 205 patients (97.6\% sensitive) with RIF-resistant bacteria and 504 of 514 (98.1\% specific) with RIF-sensitive bacteria [49 ${ }^{\bullet \bullet}$ ]. The assay is designed for direct genotypic DST from unprocessed sputum or sediment from a concentrated specimen. Sample reagent is poured into the sample tube, incubated for $15 \mathrm{~min}$, pippetted into the Xpert cartridge and inserted into the GeneXpert machine for processing. Highly trained staff are not required to run the machine or interpret the results $\left[10^{\circ}\right]$. The Xpert MTB/RIF test has the potential to be used in moderately equipped laboratories; however, it is unlikely to be used as a POC diagnostic test in the most peripheral settings, like rural health centres, due to poor infrastructure and limited resources [50]. The FIND-negotiated price for a four testing module GeneXpert machine is approximately US $\$ 17000$ and the Xpert MTB/RIF tests cost approximately US $\$ 17$ per cartridge, but the price is likely to decrease as a result of increased sales related to endorsement of the technology by the WHO in December $2010\left[10^{\bullet}, 50\right]$.

There is an urgent need for research on the implementation of the Xpert MTB/RIF test to determine its performance characteristics and the impact it has on patient outcomes when used in a variety of primary healthcare settings covering the wide range of available infrastructure. Other variables that may affect the tests overall performance are HIV prevalence, strain diversity, prevalence of specific drug resistance-conferring mutations, patient-related diagnostic delays and default rates $\left[10^{\circ}\right]$.

\section{Future prospects}

The ideal TB diagnostic would be a simple, low-technology, rapid, POC test with accurate results that could simultaneously identify drug resistance. In 2009, an expert group led by Medecins Sans Frontieres developed a set of minimum technical specifications for new POC TB tests [51]. No existing test meets all of these specifications, although the Xpert MTB/RIF test meets the majority. Increased availability of funding and growing interest in new TB diagnostics and biomarkers have encouraged the development of several new POC tests for TB including improved serologic assays, hand-held molecular devices, breath-based assays for the detection of volatile organic compounds, microchip technologies and proteomics-based and metabolomics-based tests $\left[29^{\circ}\right]$. As POC test technology advances, new POC TB and drug-resistant TB tests reach the market and competition increases, prices for such tests should reduce significantly $\left[10^{\bullet}, 50,52\right]$.

\section{Conclusion}

Current technologies for the detection of drug-resistant M. tuberculosis vary greatly in terms of turnaround time, cost and complexity. Ultimately, the 'holy grail' TB diagnostic test that fulfils all technical specifications for a good POC test may be developed. Until then, different DST methods are required, suitable for countries with diverse economic status and $\mathrm{TB}$ burden and for the various health system levels.

\section{Acknowledgements}

A.Z., J.O.G., M.B., P.M. and M.H. receive support from the EU-FP7, EDCTP, EuropeAID-ADAT, M.M. from HLF and Vinnova, Sweden as well as from EDCTP and A.Z. from the UK MRC and the UK NIHR UCLH-CBRC.

There are no conflicts of interest. 


\section{References and recommended reading}

Papers of particular interest, published within the annual period of review, have been highlighted as:

- of special interest

-• of outstanding interest

Additional references related to this topic can also be found in the Current World Literature section in this issue (p. 210).

1 Wallis RS, Pai M, Menzies D, et al. Biomarkers and diagnostics for tubercu-

- losis: progress, needs, and translation into practice. Lancet 2010; 375:1920-1937.

Comprehensive review of tuberculosis biomarkers and diagnostics.

2 Stop-TB Partnership. The global plan to stop TB 2011-2015: transforming the fight towards elimination of tuberculosis. Geneva: World Health Organisation; 2010.

3 Ellner JJ. The emergence of extensively drug-resistant tuberculosis: a global health crisis requiring new interventions. Part I: The origins and nature of the problem. Clin TransI Sci 2008; 1:249-254.

4 Ellner JJ. The emergence of extensively drug-resistant tuberculosis: a global health crisis requiring new interventions. Part II: Scientific advances that may provide solutions. Clin Transl Sci 2009; 2:80-84.

5 Fears R, Kaufmann S, Ter Meulen V, et al. Drug-resistant tuberculosis in the

- European Union: opportunities and challenges for control. Tuberculosis (Edinb) 2010; 90:182-187.

Summarizes the problem of MDR-TB in eastern Europe and the action required to tackle the problem.

6 Wright A, Zignol M, Van Deun A, et al. Epidemiology of antituberculosis drug resistance 2002-07: an updated analysis of the Global Project on AntiTuberculosis Drug Resistance Surveillance. Lancet 2009; 373:1861-1873.

7 Migliori GB, Dheda K, Centis R, et al. Review of multidrug-resistant and

-• extensively drug-resistant TB: global perspectives with a focus on subSaharan Africa. Trop Med Int Health 2010; 15:1052-1066.

Details the emerging problem of drug-resistant TB in Africa and the ominous problem of XDR-TB.

8 WHO. Multidrug and extensively drug-resistant TB (M/XDR-TB): 2010 Global

- Report on Surveillance and Response. Geneva: World Health Organisation; 2010.

Global data on drug-resistant TB surveillance.

9 Caws M, Ha DT. Scale-up of diagnostics for multidrug resistant tuberculosis. Lancet Infect Dis 2010; 10:656-658.

10 Van Rie A, Page-Shipp L, Scott L, et al. Xpert(R) MTB/RIF for point-of-care

- diagnosis of TB in high-HIV burden, resource-limited countries: hype or hope? Expert Rev Mol Diagn 2010; 10:937-946.

Article debates the importance of the latest Xpert MTB-RIF assay and whether it presents a major advance or not.

11 Martin $A$, Panaiotov $S$, Portaels $F$, et al. The nitrate reductase assay for the rapid detection of isoniazid and rifampicin resistance in Mycobacterium tuberculosis: a systematic review and meta-analysis. J Antimicrob Chemother 2008; 62:56-64.

12 WHO. Framework for Implementing New Tuberculosis Diagnostics. Geneva: World Health Organisation; 2010.

13 Lin SYG, Desmond E, Bonato D, et al. Multicenter Evaluation of BACTEC

-• MGIT 960 System for second-line drug susceptibility testing of Mycobacterium tuberculosis complex. J Clin Microbiol 2009; 47:3630-3634.

A comprehensive study of the BACTEC MGIT 960 System for second-line DST.

14 Martin A, von Groll A, Fissette K, et al. Rapid detection of Mycobacterium tuberculosis resistance to second-line drugs by use of the manual mycobacterium growth indicator tube system. J Clin Microbiol 2008; 46:39523956.

15 Visalakshi P, Meharwal SK, Myneedu VP, et al. Evaluation of direct method of drug susceptibility testing of Mycobacterium tuberculosis to rifampicin and isoniazid by nitrate reductase assay in a national reference laboratory. Diagn Microbiol Infect Dis 2010; 66:148-152.

16 Morcillo N, Imperiale B, Di Giulio B. Evaluation of MGIT 960 and the

- colorimetric-based method for tuberculosis drug susceptibility testing. Int J Tuberc Lung Dis 2010; 14:1169-1175.

Reviews the value of the MGIT 960 system for DST.

17 Minion J, Leung E, Menzies D, et al. Microscopic-observation drug suscept-

-. ibility and thin layer agar assays for the detection of drug resistant tuberculosis: a systematic review and meta-analysis. Lancet Infect Dis 2010; 10:688-698.

An overview of all data published on MODS.

18 WHO. Non-commercial culture and drug-susceptibility testing methods for screening of patients at risk of multi-drug resistant tuberculosis. Geneva: World Health Organisation; 2010.
19 Coronel J, Roper M, Mitchell S, et al. MODS accreditation process for regional reference laboratories in Peru: validation by GenoType(R) MTBDRplus. Int J Tuberc Lung Dis 2010; 14:1475-1480.

20 Brady MF, Coronel J, Gilman RH, et al. The MODS method for diagnosis of tuberculosis and multidrug resistant tuberculosis. J Vis Exp 2008; 18. http:// www.jove.com/details.stp?id=845. doi:10.3791/845.

21 Coronel J, Roper H, Caviedes L, et al. MODS: a user guide (v12.1). Microscopic observation drug susceptibility assay. http://www.modsperu.org/ MODS_user_guide.pdf [accessed 19 November 2010].

22 Affolabi D, Sanoussi N, Odoun M, et al. Rapid detection of multidrug-resistant Mycobacterium tuberculosis in Cotonou (Benin) using two low-cost colorimetric methods: resazurin and nitrate reductase assays. J Med Microbiol 2008; 57:1024-1027.

23 Farnia $\mathrm{P}$, Masjedi MR, Mohammadi F, et al. Colorimetric detection of multidrug-resistant or extensively drug-resistant tuberculosis by use of malachite green indicator dye. J Clin Microbiol 2008; 46:796-799.

24 Gupta M, Singh NP, Kaur IR. Evaluation of nitrate reductase assay for direct

- detection of drug resistance in Mycobacterium tuberculosis: rapid and inexpensive method for low-resource settings. Indian J Med Microbiol 2010; 28:363-365.

Describes a rapid and inexpensive method for DST in resource-poor countries.

25 Visalakshi P, Arora J, Behera D. Evaluation of nitrate reductase assay for rapid detection of resistance to second-line drugs in Mycobacterium tuberculosis in a tertiary care hospital. Diagn Microbiol Infect Dis 2010; 68:236-240.

26 BD. BACTEC ${ }^{\text {TM }}$ MGIT $^{\text {TM }} 960$ SIRE Kit For the antimycobacterial susceptibility testing of Mycobacterium tuberculosis. Franklin Lakes, NJ, USA: Becton Dickinson and Company; 2002.

27 WHO. Use of Liquid TB culture and drug susceptibility testing (DST) in lowand medium-income settings Geneva: World Health Organisation; 2007.

28 Piersimoni C, Olivieri A, Benacchio L, et al. Current perspectives on drug susceptibility testing of Mycobacterium tuberculosis complex: the automated nonradiometric systems. J Clin Microbiol 2006; 44:20-28.

29 Pai M, Minion J, Sohn H, et al. Novel and improved technologies for tubercu-

- Iosis diagnosis: progress and challenges. Clin Chest Med 2009; 30:701716.

A critical review of new TB diagnostics.

30 Palomino JC, Martin A, Von Groll A, et al. Rapid culture-based methods for drug-resistance detection in Mycobacterium tuberculosis. J Microbiol Methods 2008; 75:161-166.

31 Martin A, Portaels F, Palomino JC. Colorimetric redox-indicator methods for the rapid detection of multidrug resistance in Mycobacterium tuberculosis: a systematic review and meta-analysis. J Antimicrob Chemother 2006 59:175-183

$32 \mathrm{Kim} \mathrm{JH}, \mathrm{Kim}$ YJ, Ki CS, et al. Evaluation of COBAS TaqMan(R) MTB PCR for

-• the detection of Mycobacterium tuberculosis. J Clin Microbiol 2011; 49:173176.

A clinical evaluation study of an important molecular diagnostics test for TB.

33 Drews $\mathrm{S}$, Eshaghi A, Pyskir D, et al. The relative test performance characteristics of two commercial assays for the detection of Mycobacterium tuberculosis complex in paraffin-fixed human biopsy specimens. Diagn Pathol 2008; 3:37.

34 Neonakis IK, Gitti Z, Baritaki S, et al. Evaluation of GenoType mycobacteria

- direct assay in comparison with Gen-Probe Mycobacterium tuberculosis amplified direct test and GenoType MTBDRplus for direct detection of Mycobacterium tuberculosis complex in clinical samples. J Clin Microbiol 2009; 47:2601-2603.

A clinical evaluation and comparison of three important molecular diagnostics tests for TB.

35 Banerjee R, Allen J, Lin SYG, et al. Rapid drug susceptibility testing with a molecular beacon assay is associated with earlier diagnosis and treatment of multidrug-resistant tuberculosis in California. J Clin Microbiol 2010; 48:3779-3781.

36 Blakemore R, Story E, Helb D, et al. Evaluation of the analytical performance of the Xpert(R) MTB/RIF assay. J Clin Microbiol 2010; 48:2495-2501.

37 Albert $\mathrm{H}$, Bwanga F, Mukkada S, et al. Rapid screening of MDR-TB using molecular Line Probe Assay is feasible in Uganda. BMC Infect Dis 2010; 10:41.

38 Migliori GB, Matteelli $\mathrm{A}$, Cirillo $\mathrm{D}$, et al. Diagnosis of multidrug-resistant tuberculosis and extensively drug-resistant tuberculosis: current standards and challenges. Can J Infect Dis Med Microbiol 2008; 19:169-172.

39 Bwanga F, Hoffner S, Haile M, et al. Direct susceptibility testing for multi drug resistant tuberculosis: a meta-analysis. BMC Infect Dis 2009; 9:67.

40 Garza-González E, González GM, Renteria A, et al. A pyrosequencing method for molecular monitoring of regions in the inhA, ahpC and rpoB genes of Mycobacterium tuberculosis. Clin Microbiol Infect 2010; 16:607-612. 
41 Sandgren A, Strong M, Muthukrishnan $P$, et al. Tuberculosis drug resistance - mutation database. PLoS Med 2009; 6:e1000002.

A valuable database containing information on $M$. tuberculosis drug resistance conferring mutations.

42 WHO. Molecular line probe assays for rapid screening of patients at risk of multidrug-resistant tuberculosis (MDR-TB). Geneva: World Health Organisation; 2008.

43 Ling DI, Zwerling AA, Pai M. GenoType MTBDR assays for the diagnosis of multidrug-resistant tuberculosis: a meta-analysis. Eur Respir J 2008; 32:1165-1174.

44 Kiet VS, Lan NTN, An DD, et al. Evaluation of the MTBDRsl test for detection of second-line-drug resistance in Mycobacterium tuberculosis. J Clin Microbiol 2010; 48:2934-2939.

45 Hillemann D, Rusch-Gerdes S, Richter E. Feasibility of the GenoType(R) MTBDRsl assay for fluoroquinolone, amikacin/capreomycin, and ethambutol resistance testing of Mycobacterium tuberculosis strains and in clinical specimens. J Clin Microbiol 2009; 47:1767-1772.

46 Brossier F, Veziris N, Aubry A, et al. Detection by GenoType MTBDRsl test of complex mechanisms of resistance to second-line drugs and ethambutol in multidrug-resistant Mycobacterium tuberculosis complex isolates. J Clin Microbiol 2010; 48:1683-1689.
47 Nicol MP. New developments in the laboratory diagnosis of tuberculosis. Continu Med Educ 2010; 28:246-250.

48 Helb D, Jones M, Story E, et al. Rapid detection of Mycobacterium tubercu- Iosis and rifampicin resistance by use of on-demand, near-patient technology. J Clin Microbiol 2010; 48:229-237.

First evaluation of the Xpert MTB-RIF assay.

49 Boehme CC, Nabeta P, Hillemann D, et al. Rapid molecular detection of - tuberculosis and rifampicin resistance. N Engl J Med 2010; 363:10051015.

Large multicentre study evaluating the use of Xpert MTB-RIF assay at points of care.

50 Lemaire JF, Casenghi M. New diagnostics for tuberculosis: fulfilling patient needs first. J Int AIDS Soc 2010; 13:40.

51 Medecins Sans Frontieres. Defining test specifications for a TB POC test. http://www.msfaccess.org/TB_POC_Parismeeting/ [accessed November 2010].

52 Sohn $\mathrm{H}$, Minion J, Albert $\mathrm{H}$, et al. TB diagnostic tests: how do we figure out their costs? Expert Rev Anti Infect Ther 2009; 7:723-733. 